\title{
Ashy dermatosis with involvement of mucous membranes*
}

\author{
Margarida Rato ${ }^{1}$ \\ João Aranha ${ }^{1}$
}

\author{
Ana Filipe Monteiro ${ }^{1}$ \\ Ermelindo Tavares ${ }^{1}$
}

DOI: http:/ / dx.doi.org/10.1590/abd1806-4841.20176133

\begin{abstract}
Ashy dermatosis is a rare condition, of unknown aetiology, in which mucous membranes are typically spared. The authors report the case of a 57-year-old female with a history of asymptomatic gray-bluish macules located on the trunk and oral mucosa. There were no relief changes on examination. Skin biopsies from the oral mucosa and trunk were performed and both were compatible with ashy dermatosis. The patient started treatment with oral clofazimine but due to the absence of clinical improvement the drug was discontinued three months later. This case report illustrates an atypical case of ashy dermatosis owing to the involvement of mucous membranes, which is rarely described in the literature.
\end{abstract}

Kewbords: Hyperpigmentation; Clofazimine; Oral Mucosa

\section{INTRODUCTION}

Ashy dermatosis (AD), or erythema dyschromicum perstans, was first described in 1957 by Ramirez in Salvadorans. ${ }^{1}$ It is a relatively rare, idiopathic, acquired disorder, that tends to be more prevalent in Central and South America, although there are cases reported from different regions of the world. ${ }^{2-4} \mathrm{AD}$ usually appears in adults - even though there are reports in children - with no clear gender preference., ${ }^{3,4}$ Higher phototypes are more frequently affected. ${ }^{2-5}$

\section{CASE REPORT}

A 57-year-old Caucasian female, phototype III, reported a history of asymptomatic lesions on the oral mucosa and trunk. The lesions were present for 5 years, with progressive worsening over the first 12 months, and had not been preceded by any other changes. The lesions on the trunk appeared over the past 4 months, with increase in number and size in the first 3 months. Such as on the oral mucosa, these lesions were not preceded by any skin changes. The patient denied taking new medications or previous infectious diseases before the appearance of the lesions. In her past history, there was hypothyroidism, hypertension and dyslipidemia, and she was being medicated with lisinopril and hydrochlorothiazide $(20 \mathrm{mg} /$ day and $12.5 \mathrm{mg} /$ day) and simvastatin (20mg/day).

On dermatological examination, there were oval-shaped, blue-grey, confluent ill-defined macules, with $1 \mathrm{~cm}$ to $2 \mathrm{~cm}$ in their larger diameter, on the lumbar region, right hypochondrium, right iliac fossa, labial mucosa, lateral borders of the tongue and jugal mucosa (Figures 1 to 4 ). There were no erythematous borders. The rest of the examination was normal.

Blood count, liver, renal and thyroid function tests, urinalysis, feces parasitology and serologies (including syphilis, hepatitis

Study submitted on 07.06.2016

Approved by the Advisory Board and accepted for publication on 09.10.2016

* Study conducted at Hospital de Santarém, EPE - Santarém, Portugal.

Financial Support: None.

Conflict of Interests: None.

1 Derpatment of Dermatology at Hospital de Santarém, EPE - Santarém, Portugal.

()2017 by Anais Brasileiros de Dermatologia 
C, hepatitis B and HIV infection) were all within normal limits or negative. Histopathology of a skin sample taken from the lumbar region demonstrated mild acanthosis and follicular hyperkeratosis, mild lymphohistiocytic inflammatory infiltrate in the superficial dermis and melanophages (Figure 5). Histopathology from a mucosal sample showed mild to moderate regular acanthosis and lymphohistiocytic infiltrate in the superficial lamina propria with

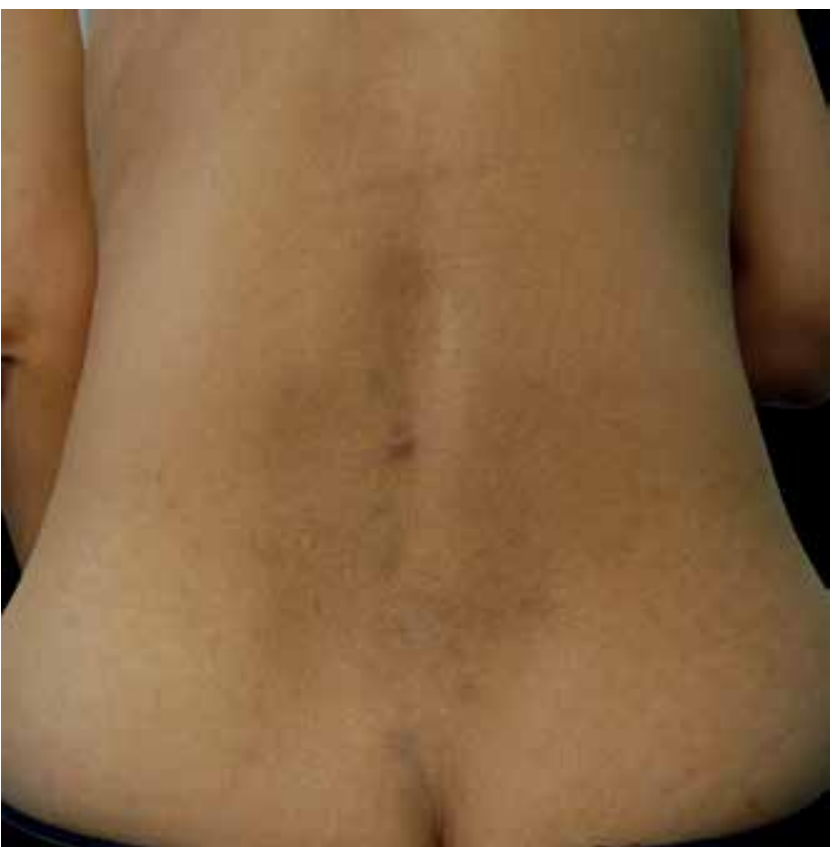

Figure 1: Blue-grey, ill-defined, oval-shaped macules on the lumbar region

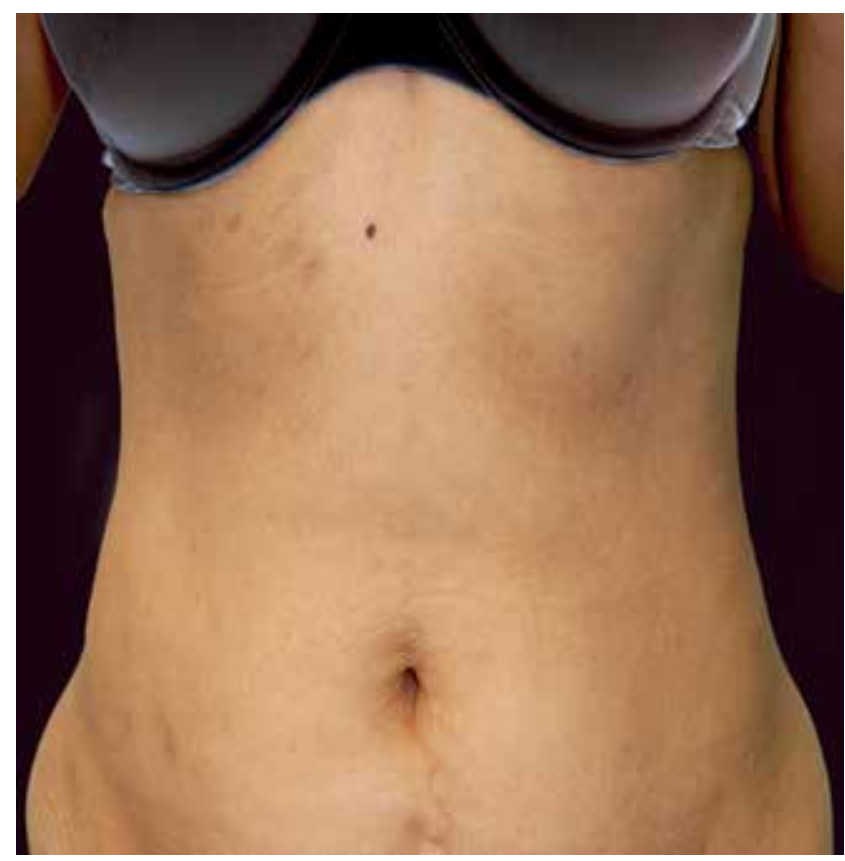

Figure 2: Blue-grey, ill-defined, oval-shaped macules on the right hypochondrium and right iliac fossa numerous melanophages (Figure 6). The combination of the clinical findings with laboratory tests and histopathology lead to the diagnosis of AD.

Oral clofazimine (100mg three times per week) was commenced and, after 3 months, there was no clinical improvement; the patient opted to discontinue the treatment.

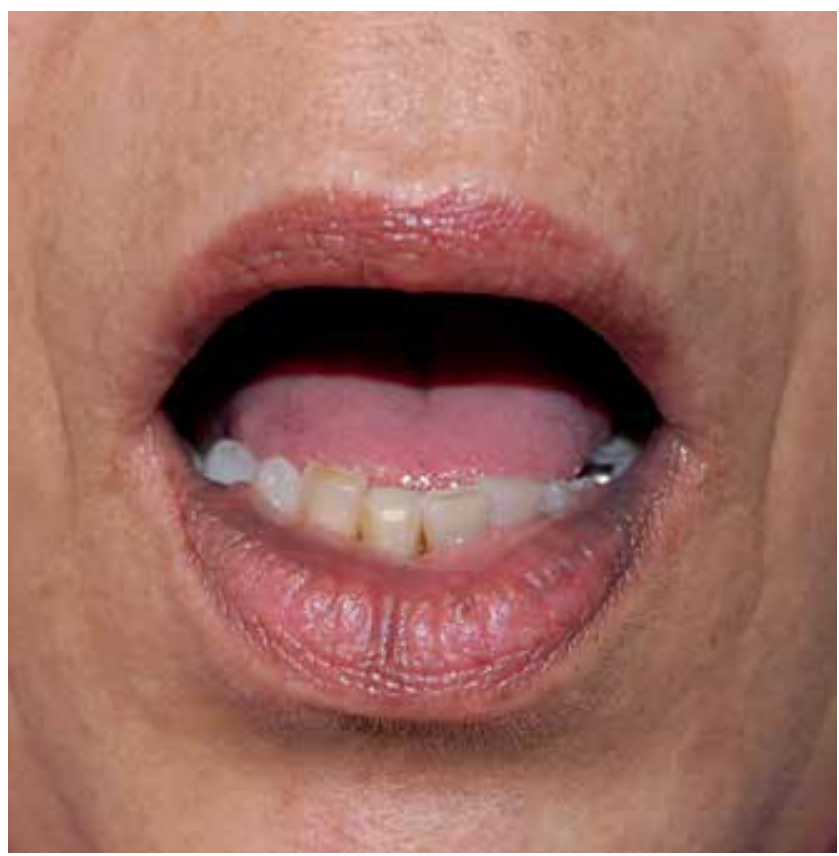

Figure 3: Blue-grey macules on the labial mucosa

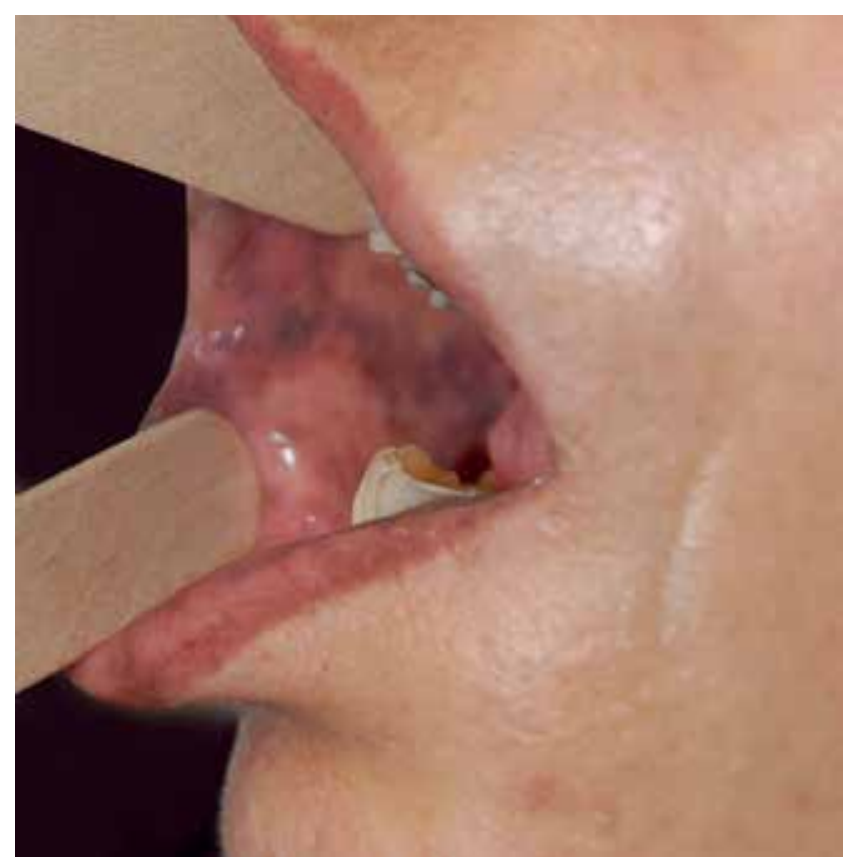

Figure 4: Blue-grey macules on the jugal mucosa 


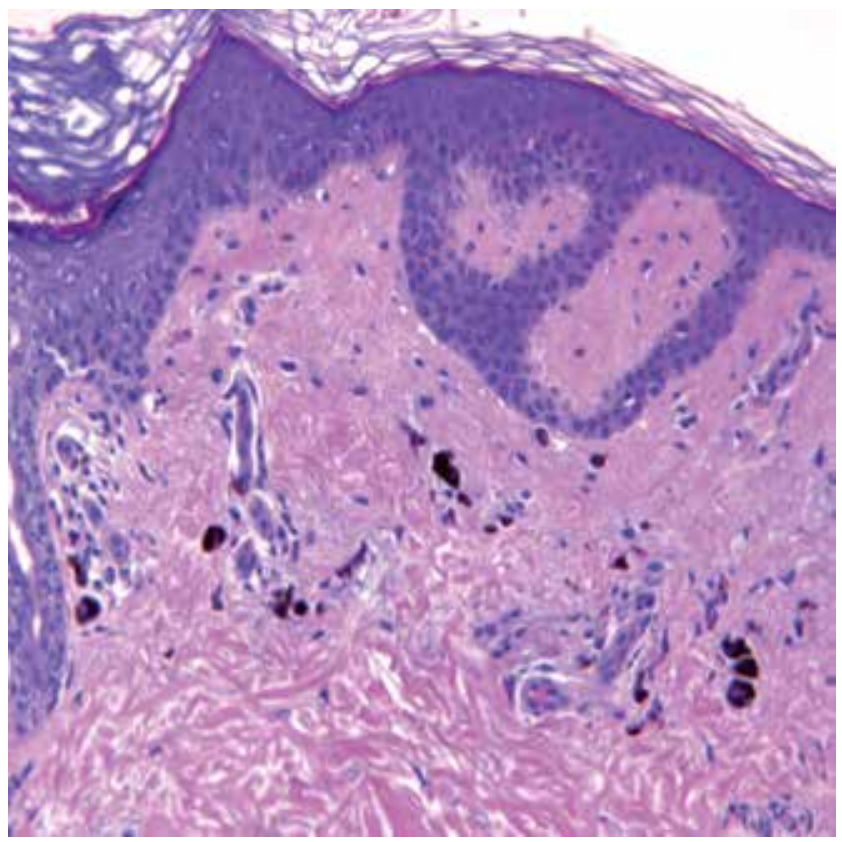

Figure 5: Histopathology of the lumbar lesion. Mild acanthosis and mild lymphohistiocytic inflammatory infiltrate in the superficial dermis with melanophages (Hematoxylin \& eosin, X100)

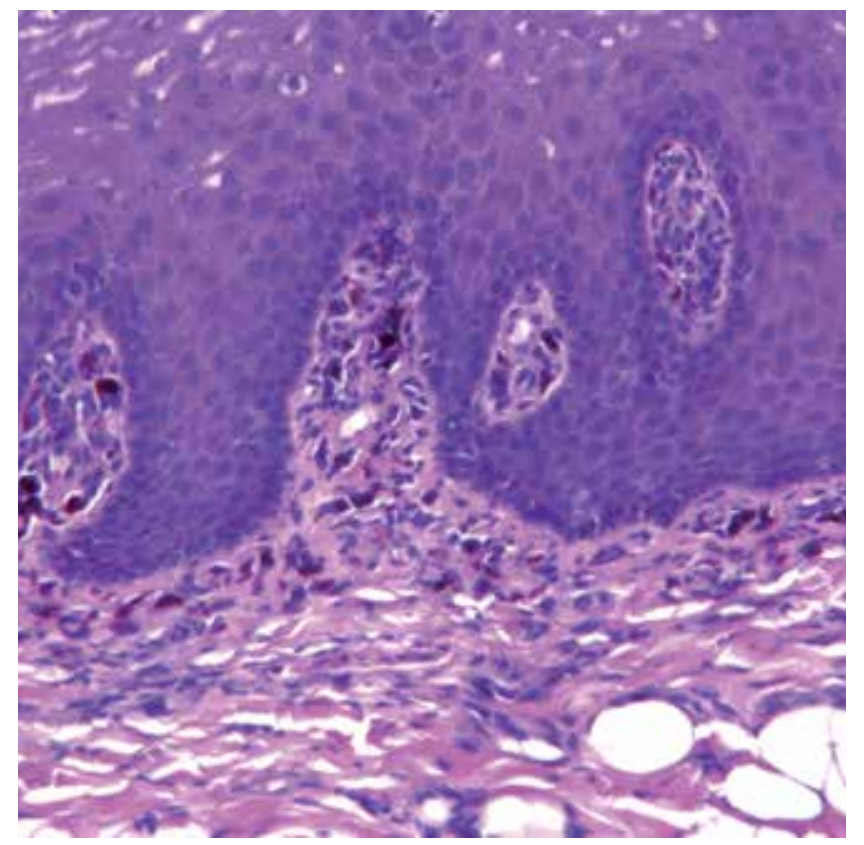

Figure 6: Histopathology of the lesion on the jugal mucosa. Lymphohistiocytic infiltrate in the superficial lamina propria with numerous melanophages (Hematoxylin \& eosin, X400)

\section{DISCUSSION}

$\mathrm{AD}^{\prime}$ 's etiology is still unknown. There are, however, isolated case reports associated to endocrine diseases (thyroid pathology), drugs (ethambutol, penicillin and benzodiazepines), ingestion of toxins (ammonium nitrate, cobalt, contrast media, fungicides, pesticides), infections (HIV, hepatitis $\mathrm{C}$ virus, Trichuris trichiura) and vitiligo. ${ }^{2-4}$ The identification of the allelic subtype HLA-DR4*0407 is a risk factor in Mexican mestizos. ${ }^{5}$ In virtue of the presence of interleukins and inflammatory mediators in the lesions, some studies indicate that this condition might be immunomediated, probably in association with a genetic predisposition. ${ }^{3,5,6}$ In this case, the fact that the patient had past history of hypothyroidism supports a possible association between both conditions, such as described in the literature.

$\mathrm{AD}$ is characterized by the progressive and symmetric development of grey, oval-shaped or polycyclic asymptomatic macules, mainly on the trunk and proximal extremities, but also on the cervical region and face..$^{2-5,7}$ The lesions can have an erythematous border initially, slightly raised, with a diameter ranging from $3 \mathrm{~mm}$ and many centimeters, that can eventually disappear after a few months. ${ }^{2-5,7}$ The lesions can become confluent and affect almost the whole skin, with no preference for photoexposed areas or not. ${ }^{2-4} \mathrm{Pal}-$ mar and plantar regions, scalp, nails and mucous membranes are typically spared., ${ }^{3,7}$ To the authors knowledge, this is the first case reported in which $\mathrm{AD}$ affects the mucous membranes.

Histopathology is not diagnostic for AD. A perivascular lymphohistiocytic inflammatory infiltrate in the dermis, multiple melanophages in the papillary dermis with pigmentary incontinence, lymphocyte exocytosis, colloid bodies and vacuolar degeneration of the basal cells can be seen. ${ }^{4,7}$, Torrelo et al. ${ }^{4}$ described a series of 14 children with AD whose skin biopsies revealed different histopathology, with different combinations of vacuolar changes, colloid bodies, perivascular infiltrates and melanophages. In all cases, marked pigmentary incontinence and perivascular infiltrates were seen, however, lichenoid changes were observed in only a few. These authors suggest that the lichenoid changes could be restricted to the periphery of the lesion and, as such, cannot be identified if this area is not included in the biopsy or if multiple sections are not examined. ${ }^{4}$

The differential diagnosis of $\mathrm{AD}$ includes lichen planus pigmentosus (LPP), lichenoid drug reactions, pigmented fixed erythema, post-inflammatory hyperpigmentation, idiopathic eruptive macular pigmentation, urticaria pigmentosa, Addison's disease and hemochromatosis. ${ }^{2-4,8}$ In fact, AD is a controversial entity, since some authors consider it a variant of LPP due to the overlap of clinical findings and histopathology, ${ }^{4,7}$ However, there are significant clinical differences between both conditions, allowing them to be classified as distinct entities. ${ }^{3,4}$ LPP is characterized by brown, itchy macules and papules, sometimes in a reticular pattern, with no active erythematous border, on photoexposed areas and flexures; it is chronic, with recurrences and remissions. ${ }^{3,9}$ In the case described, the fact that the condition has an insidious progression, with bluegrey, asymptomatic, relatively symmetrical macules without a reticular pattern on non-photoexposed areas, and the absence of flexural lesions supports the diagnosis of AD, instead of LPP. 
$\mathrm{AD}$ is usually a chronic condition, and spontaneous remission is rare in adults. ${ }^{2,4,5,7}$ Different drugs have been suggested for the treatment, but the results have not been consistent. There are no prospective, randomized, controlled studies to serve as therapeutic guide. ${ }^{4,5,10}$ Some of the options include dapsone, oral and topical corticosteroids, antibiotics, antihistamines, isoniazid, griseofulvin, antimalarials, keratolytic agents and phototherapy, with variable efficacy and recurrence rates..$^{2-5,7,8,10}$ Clofazimine has been used with a response rate of $66 \%$ to $78 \% .^{2}$ Its beneficial effect could be mediated by an anti-inflammatory and immunomodulating effect. ${ }^{3,10}$ Besides, by causing a homogenous coloration of the skin, therefore masking the lesions, it also has a cosmetic effect, which can also explain its efficacy. ${ }^{2-4,10} \square$

\section{REFERENCES}

1. Ramirez C0. Los Cenicientos: problema Clínico. In: Proceedings of the first Central American Congress of Dermatology. San Salvador, December $5-8$, 1957, p. $122-130$.

2. Tlougan BE, Gonzalez ME, Mandal RV, Kundu RV, Skopicki DL. Erythema dyschromicum perstans. Dermatol Online J. 2010;16:17.

3. Schwartz RA. Erythema dyschromicum perstans: the continuing enigma of Cinderella or ashy dermatosis. Int J Dermatol. 2004:43:230-2.

4. Torrelo A, Zaballos P, Colmenero I, Mediero IG, de Prada I, Zambrano A. Erythema dyschromicum perstans in children: a report of 14 cases. J Eur Acad Dermatol Venereol. 2005;19:422-6.

5. Correa MC, Memije EV, Vargas-Alarcón G, Guzmán RA, Rosetti F, Acuña-Alonzo $\mathrm{V}$, et al. HLA-DR association with the genetic susceptibility to develop ashy dermatosis in Mexican Mestizo patients. J Am Acad Dermatol. 2007;56:617-20.

6. Vásquez-Ochoa LA, Isaza-Guzmán DM, Orozco-Mora B, Restrepo-Molina R, Trujillo-Perez J, Tapia FJ. Immunopathologic study of erythema dyschromicum perstans (ashy dermatosis). Int J Dermatol. 2006;45:937-41.

7. Novick NL, Phelps R. Erythema dyschromicum perstans. Int J Dermatol. 1985;24:630-3.

8. Zaynoun S, Rubeiz N, Kibbi AG. Ashy dermatosis - a critical review of the literature and a proposed simplied clinical classication. Int J Dermatol. 2008;47:542-4.

9. Vega ME, Waxtein L, Arenas R, Hojyo T, Dominguez-Soto L. Ashy dermatosis and lichen planus pigmentosus: a clinicopathologic study of 31 cases. Int J Dermatol. 1992;31:90-4.

10. Piquero-Martín J, Pérez-Alfonzo R, Abrusci V, Briceño L, Gross A, Mosca W, et al. Clinical trial with clofazimine for treating erythema dyschromicum perstans. Evaluation of cell-mediated Immunity. Int J Dermatol. 1989;28:198-200.
MAILING ADDRESS:

Margarida Pinheiro Gaio Seabra Rato

Serviço de Dermatologia do Hospital de Santarém

Avenida Bernardo Santareno

2005-177 Santarém, Portugal

E-mail:margaridagaiorato@gmail.com

How to cite this article: Rato M, Monteiro AF, Aranha J, Tavares E. Ashy dermatosis with involvement of mucous membranes. An Bras Dermatol. 2017;92(5 Suppl 1):17-20. 